\title{
ESTUDO PARA RECUPERAÇÃO DO REJEITO FINAL DA FLOTAÇÃO*
}

\author{
Anderson William Henrique Satini ${ }^{1}$ \\ João Paulo Sousa Coelho' \\ Wellington Ribeiro Moreira ${ }^{3}$ \\ Adriana Baldessin Costa ${ }^{4}$ \\ Wesley Paulino ${ }^{5}$
}

\section{Resumo}

A redução do preço de minério de ferro aliada ao aumento das restrições ambientais para licenciamento de áreas para disposição final de rejeito vêm impondo a necessidade de se aumentar a eficiência dos processos de concentração mineral. Dentro desse contexto, realizou-se esse trabalho com o objetivo de avaliar a viabilidade técnica de se flotar o atual rejeito final da planta de flotação da Usina Central de beneficiamento da Mina Casa de Pedra, a fim de aumentar a recuperação mássica e metálica da planta de concentração. Examinando os resultados dos ensaios laboratoriais de flotação, realizados com a amostra do atual rejeito final da concentração, foi possível constatar uma redução de $62 \%$ nos teores de ferro do rejeito flotado. Além disso, as análises estatísticas revelaram ser dispensável a dosagem suplementar de reagentes para flotar esse material.

Palavras-chave: Flotação; Rejeito; Minério de ferro.

\section{STUDY TO RECOVER THE FINAL TAILING OF FLOTATION}

\section{Abstract}

The reduction of Iron Ore's price allied to the increase of environmental restriction to get legal permission to dispose of tailings has been imposed the need to improve the efficiency of concentration process. In this context, this work evaluates technical viability of retreating final tailing of flotation columns of Mina Casa de Pedra in order to increase the metallurgical and mass recoveries. Examining results of flotation tests with sample of current final tailing it was possible to verify a reduction of $62 \%$ in the grade of iron that was floated. Moreover, statistical analyze of experimental data revealed that dosage of reactants didn't influence the final results.

Keywords: Flotation; Tailing; Iron ore.

1 Administrador de Empresas, Especialista em Engenharia de Produção, Coordenador de Controle Operacional, Gerência de Controle Operacional de Processos (GCOP), CSN (Companhia Siderúrgica Nacional), Congonhas, Minas Gerais e Brasil.

2 Engenheiro Químico, Engenheiro de Desenvolvimento, Gerência de Controle Operacional de Processos (GCOP), CSN (Companhia Siderúrgica Nacional), Congonhas, Minas Gerais e Brasil.

3 Engenheiro de Minas, Mestre em Tecnologia Mineral, Gerente de Controle Operacional de Processos, Gerência de Controle Operacional de Processos (GCOP), CSN (Companhia Siderúrgica Nacional), Congonhas, Minas Gerais e Brasil.

4 Engenheira de Minas, MBA em Estratégia Empresarial, Coordenadora de Processos, Gerência de Controle Operacional de Processos (GCOP), CSN (Companhia Siderúrgica Nacional), Congonhas, Minas Gerais e Brasil.

5 Engenheiro de Minas, Engenheiro de Processos, Gerência de Controle Operacional de Processos (GCOP), CSN (Companhia Siderúrgica Nacional), Congonhas, Minas Gerais e Brasil. 


\section{INTRODUÇÃO}

A exaustão dos depósitos de maior teor em ferro, como as reservas de hematita, impõe a necessidade de se explotar jazidas cada vez mais pobres e mais complexas, compostas majoritariamente por itabiritos, constituídos por quartzo e hematita, os quais necessitam de serem submetidos a processos de concentração antes de serem comercializados.

A liberação entre o quartzo e os minerais de ferro ocorre em frações granulométricas mais finas, característica que impõe uma grande dificuldade para separá-los, uma que vez à medida que decresce o tamanho das partículas, menos acentuada se torna a diferença existente entre as suas respectivas propriedades físicas, aumentando a susceptibilidade delas ao arraste hidrodinâmico, reduzindo o grau de separabilidade.

Esse fato é evidenciado nas características granuloquímicas do rejeito final da flotação, em Casa de Pedra. Conforme demonstrado na Figura 01, mais de $80 \%$ do ferro presente no overflow da etapa rougher, que, atualmente, consiste no rejeito final do processo de concentração, é constituído por partículas cujo tamanho é inferior a $38 \mu \mathrm{m}$. Portanto, o aumento da recuperação metálica impõe a necessidade de se aumentar o índice de recuperação dessa classe de partícula.

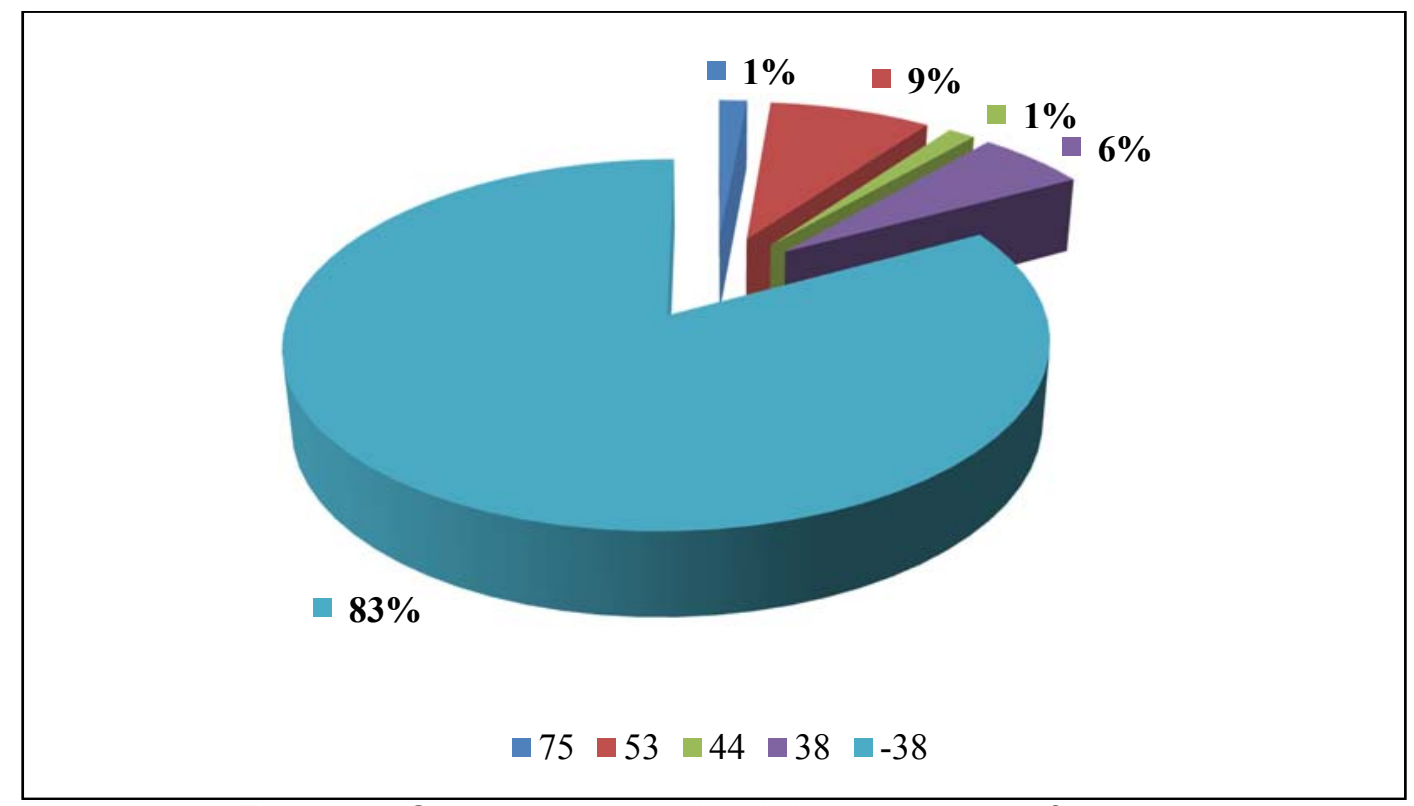

Figura 01: Composição granulométrica do rejeito da flotação

Dentro deste contexto foi feito uma série de testes laboratoriais com amostras do overflow das colunas rougher visando estudar a viabilidade técnica de se submeter o rejeito final atual a mais uma etapa de flotação, Scavenger. Os testes realizados foram previamente planejados utilizando a metodologia estatística "Planejamento Fatorial", a qual permitiu avaliar o impacto da introdução de mais uma etapa assim como a necessidade de se dosar reagentes químicos para flotar este material. 


\section{MATERIAIS E MÉTODOS}

\subsection{Planejamento Experimental}

Para realizar os ensaios, o primeiro passo foi planejar os testes laboratoriais, adotando a metodologia estatística de Planejamento Fatorial, que consiste em uma técnica que permite planejar o número e a sequência dos ensaios de forma a estudar diversos fatores, variáveis críticas do processo, em diferentes níveis, possibilitando determinar o melhor ajuste dos parâmetros operacionais (variáveis) de forma a otimizar os resultados do processo.

No trabalho em questão, as variáveis estudadas foram as dosagens de amina e amido, avaliadas em três níveis distintos: mínimo, intermediário e máximo, conforme apresentado na Tabela 1. Os índices avaliados foram o índice de recuperação metalúrgica e o teor de ferro no rejeito. Os testes foram feitos em duplicata a fim de se avaliar a significância estatística da diferença entre os resultados experimentais.

Tabela 1:Planejamento Fatorial dos ensaios de flotação.

\begin{tabular}{|c|c|c|c|c|c|c|}
\hline \multirow{2}{*}{ TESTES } & \multicolumn{3}{|c|}{ AMINA } & \multicolumn{3}{|c|}{ AMIDO } \\
\hline & 0 & 10 & 20 & 0 & 200 & 400 \\
\hline $1^{\mathrm{a}}$ & $x$ & - & - & $x$ & - & - \\
\hline $2^{\mathrm{a}}$ & $x$ & - & - & - & $x$ & - \\
\hline $3^{a}$ & $x$ & - & - & - & - & $x$ \\
\hline $4^{\mathrm{a}}$ & - & $x$ & - & $x$ & - & - \\
\hline $5^{a}$ & - & $x$ & - & - & $x$ & - \\
\hline $6^{a}$ & - & $x$ & - & - & - & $x$ \\
\hline $7^{\mathrm{a}}$ & - & - & $x$ & $x$ & - & - \\
\hline $8^{a}$ & - & - & $x$ & - & $x$ & - \\
\hline $9^{\mathrm{a}}$ & - & $x$ & - & - & - & $x$ \\
\hline $10^{\mathrm{a}}$ & $x$ & - & - & $x$ & - & - \\
\hline $11^{\mathrm{a}}$ & $x$ & - & - & - & $x$ & - \\
\hline $12^{\mathrm{a}}$ & $x$ & - & - & - & - & $x$ \\
\hline $13^{\mathrm{a}}$ & - & $x$ & - & $x$ & - & - \\
\hline $14^{\mathrm{a}}$ & - & $x$ & - & - & $x$ & - \\
\hline $15^{\mathrm{a}}$ & - & $x$ & - & - & - & $x$ \\
\hline $16^{\mathrm{a}}$ & - & - & $x$ & $x$ & - & - \\
\hline $17^{\mathrm{a}}$ & - & - & $x$ & - & $x$ & - \\
\hline $18^{\mathrm{a}}$ & - & - & $x$ & - & - & $x$ \\
\hline
\end{tabular}

\subsection{Materiais e Métodologia}

Para realização dos ensaios laboratoriais, amostrou-se o overflow das colunas rougher, retirando aproximadamente 100 litros de polpa do rejeito final da flotação.

Antes de iniciar os testes de flotação, retiraram-se duas alíquotas da amostra bruta, previamente homogeneizada, para determinar a fração mássica de sólidos assim como sua distribuição granulométrica.

Com base na fração mássica de sólidos da amostra e no volume necessário para realização dos ensaios de flotação, determinou-se a quantidade de reagentes (amina, amido e hidróxido de sódio) necessária tendo em vista as dosagens específicas ( $\mathrm{g} / \mathrm{t})$ previamente estabelecidas no planejamento fatorial (Tabela 1$)$. 
Para solubilizar o amido (Gritz de milho), empregou-se uma solução de hidróxido de sódio, sendo a razão de dosagem, em relação ao amido, igual 12/1. Já a amina utilizada foi a EDA 3C, previamente diluída a 3\% em solução aquosa.

Os ensaios de flotação foram realizados utilizando uma célula de flotação. A Tabela 2 apresenta as variáveis operacionais praticadas durante os ensaios.

Tabela 2: Parâmetros operacionais adotados durante os ensaios de flotação.

\begin{tabular}{|c|c|}
\hline Tempo de condicionamento do Amido & 1 minuto \\
\hline Tempo de condicionamento da amina & 30 segundos \\
\hline pH de trabalho & 9,46 \\
\hline Rotação do impelidor & $850 \mathrm{rpm}$ \\
\hline Tempo de Flotação & 150 segundos \\
\hline
\end{tabular}

\subsection{Caracterização da Amostra}

A Tabela 3 apresenta as características granuloquímicas da amostra estudada. Conforme abordado anteriormente e demonstrado na Tabela 3, verifica-se um aumento significativo do teor de ferro na fração granulométrica mais fina do rejeito final da flotação.

Tabela 3: Resultados granuloquímicos da amostra estudada.

\begin{tabular}{|c|c|c|c|c|c|c|c|}
\hline Malha (mm) & Retido (\%) & Pass. Ac. (\%) & $\mathbf{F e}(\%)$ & $\mathbf{S i O}_{2}(\%)$ & $\mathbf{A l}_{2} \mathbf{O}_{3}(\%)$ & $\mathbf{P}(\%)$ & $\mathbf{M n}(\%)$ \\
\hline $\mathbf{0 , 2 1 2}$ & 0,00 & 100,00 & 0,00 & 0,00 & 0,00 & 0,00 & 0,00 \\
\hline $\mathbf{0 , 1 5 0}$ & 5,91 & 94,09 & 3,12 & 95,66 & 0,39 & 0,01 & 0,03 \\
\hline $\mathbf{0 , 0 7 5}$ & 30,49 & 63,60 & 1,62 & 97,41 & 0,28 & 0,01 & 0,02 \\
\hline $\mathbf{0 , 0 4 4}$ & 25,82 & 37,77 & 6,86 & 89,37 & 0,49 & 0,01 & 0,02 \\
\hline $\mathbf{- 0 , 0 4 4}$ & 37,77 & 0,00 & 52,03 & 22,91 & 0,68 & 0,03 & 0,15 \\
\hline \multicolumn{2}{|c|}{ Global } & - & 22,10 & 67,09 & 0,49 & 0,02 & 0,07 \\
\hline \multicolumn{2}{|c|}{ Alim. Flot. } & - & 22,69 & 66,50 & 0,56 & 0,02 & 0,05 \\
\hline
\end{tabular}

\section{RESULTADOS E DISCUSSÃO}

A Tabela 4 apresenta os resultados obtidos em cada ensaio e em sua respectiva réplica, ao passo que a Figura 2 mostra um gráfico comparando a média do teor de ferro do rejeito final da flotação (amostra inicial dos testes) e a média obtida após os ensaios. Examinando a Figura 2, constata-se uma redução substancial do teor de ferro do rejeito após a realização dos testes. 


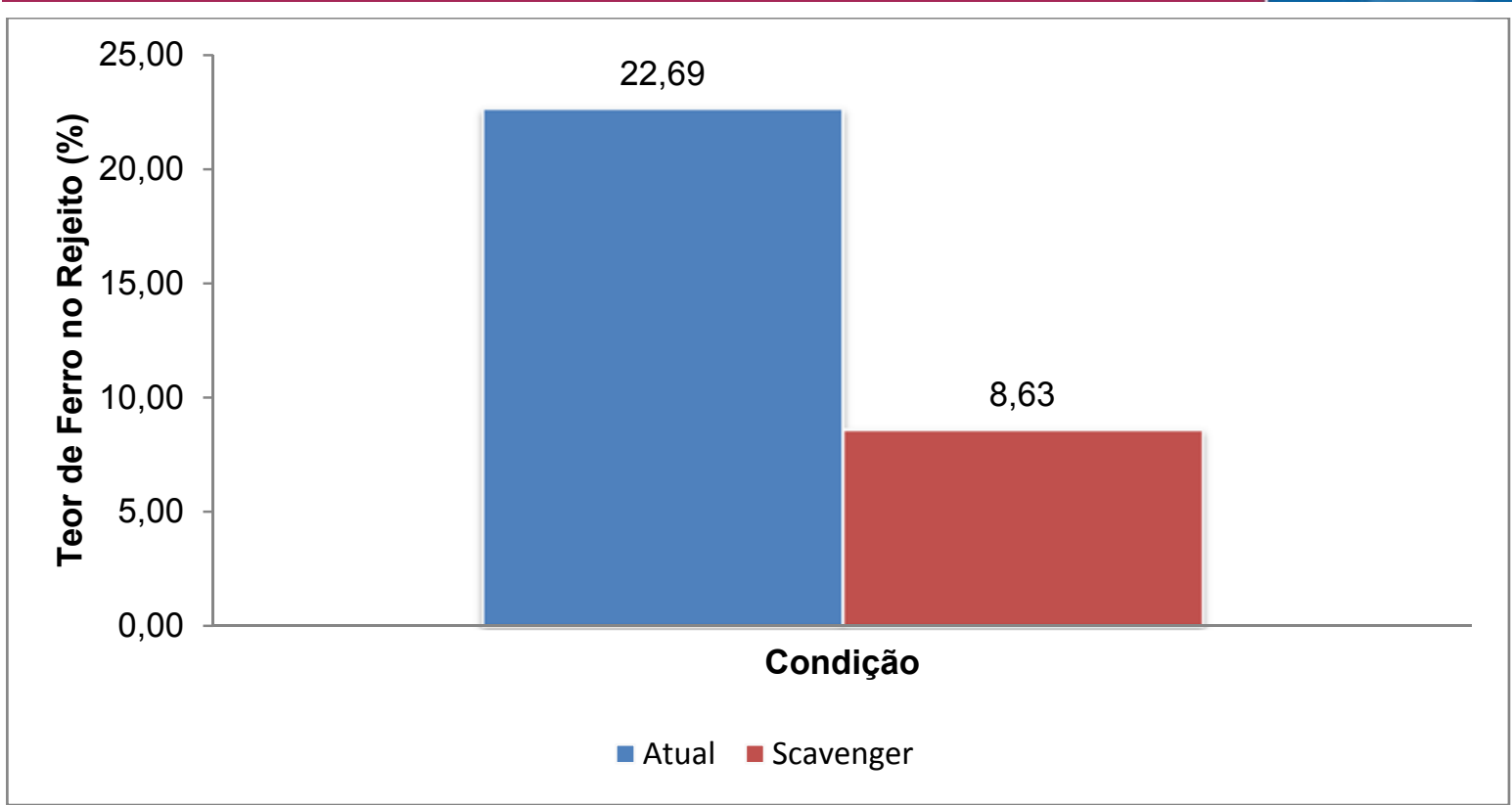

Figura 2: Comparação entre o teor de ferro do rejeito final atual da flotação e aquele obtido após os ensaios de flotação no laboratório.

Tabela 4: Resultados dos ensaios de Flotação.

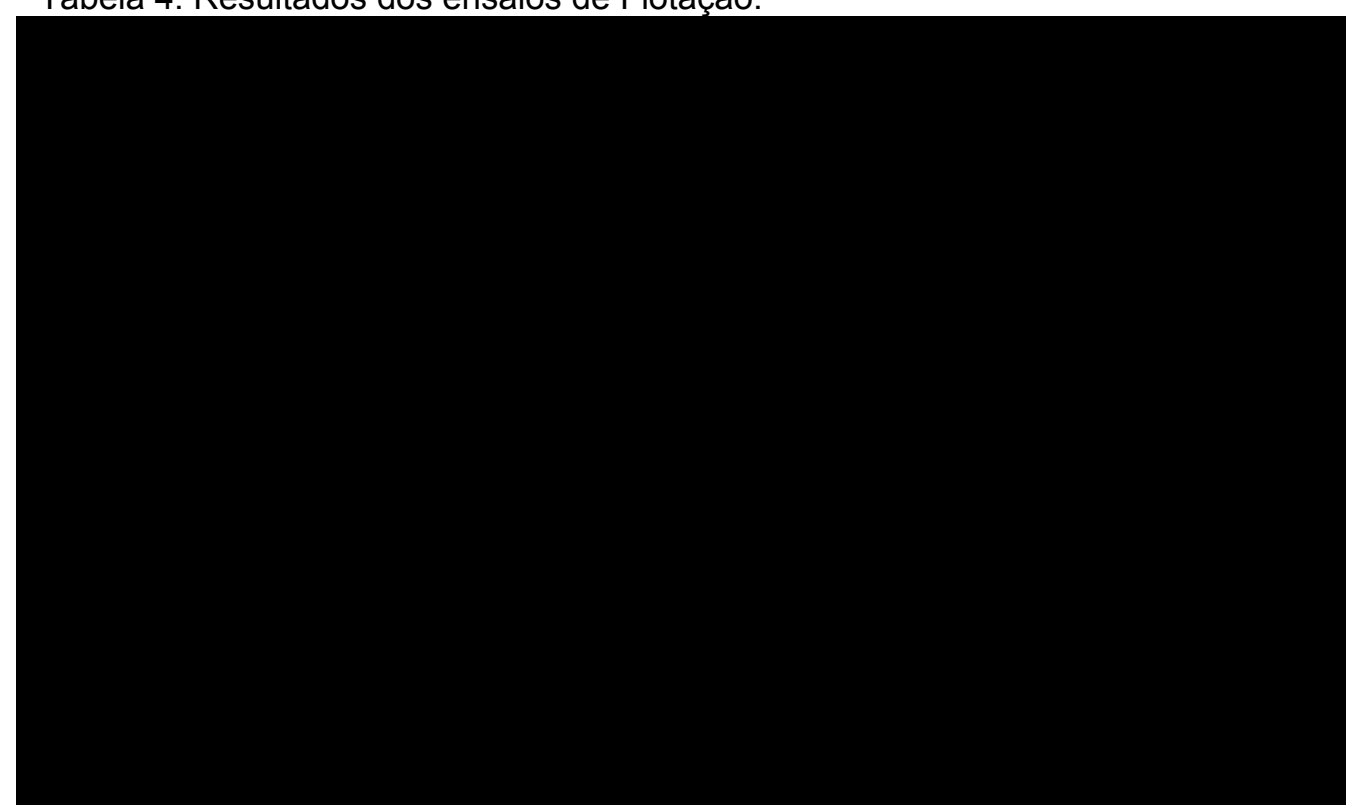

As Figuras 3 e 4 mostram os gráficos com a análise estatística contendo os resíduos dos resultados. Verifica-se, através do "Normal Probability Plot" e do "Histogram", que os resíduos são normais e centrados em zero. Já nos gráficos "Residual Versus Fits" e "Residual Versus Order" pode-se verificar que os pontos (resíduos) se distribuíram aleatoriamente ao longo dos ensaios. Os dois fatos citados evidenciam que não houve "interferências externas" durante a realização dos ensaios [1]. 


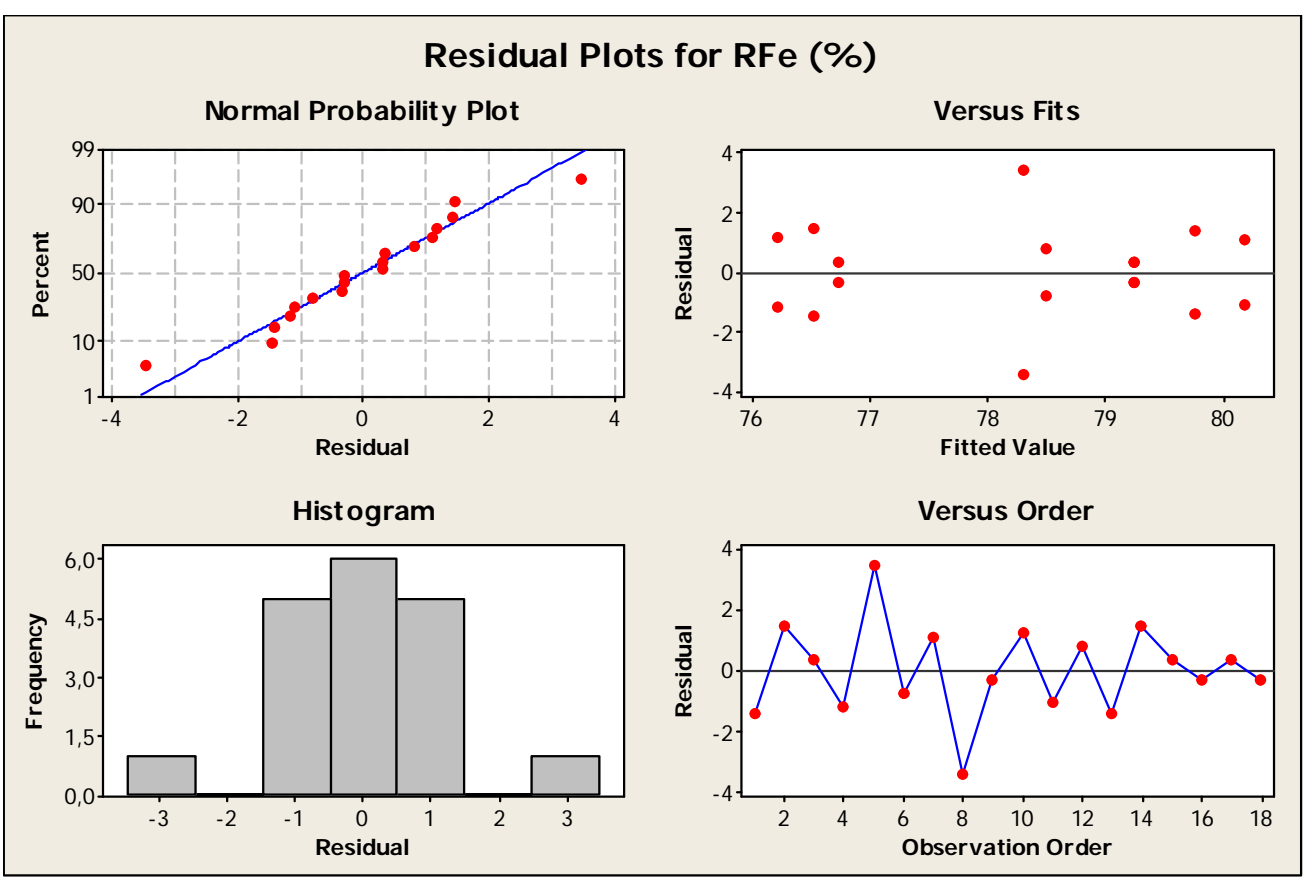

Figura 3: Gráfico com a análise estatística dos resíduos.

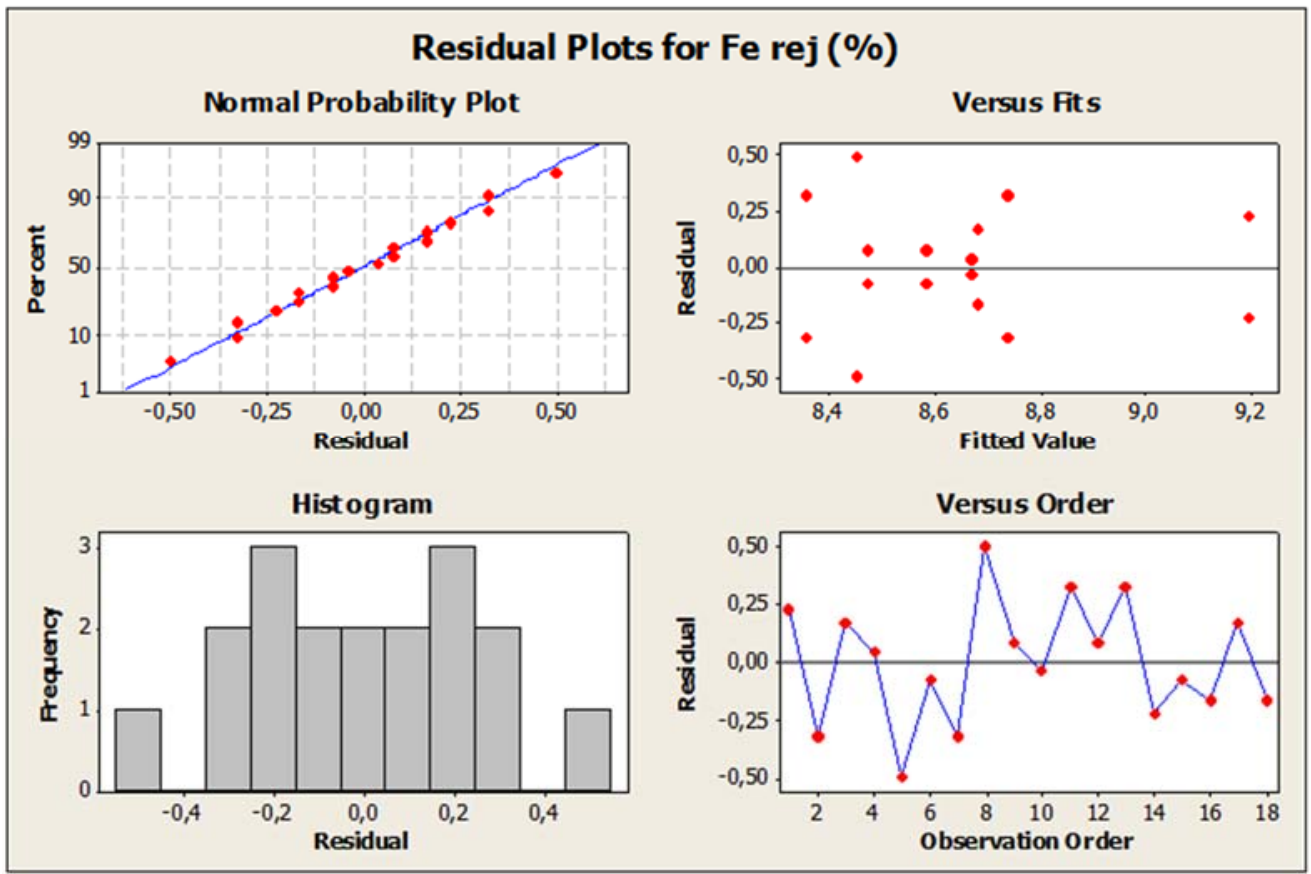

Figura 4: Gráfico com a análise estatística dos resíduos.

As Figura 5 e 6 apresentam a análise da significância estatística das variações observadas nos índices correspondentes ao teor de ferro no rejeito e à recuperação metalúrgica obtida nos ensaios. Verifica-se, analisando os valores do "P Value", cujos valores foram superiores a 0,05 (nível mínimo de significância estatística), que a dosagem dos reagentes (amido e amina) não exerceu influência significativa para a variação observada nos índices estudados [1]. Esse fenômeno pode estar relacionado ao fato de que as partículas dos minerais, presentes na amostra analisada, já terem sido previamente condicionadas com os reagentes (amido e amina), os quais são adicionados à polpa antes da etapa rougher na planta industrial e, portanto, já possuírem suas respectivas superfícies recobertas, impedindo ou dificultando nova adsorção. 


\section{General Linear Model: Fe rej (\%) versus Amina; Amido}

\begin{tabular}{|c|c|c|c|c|c|c|c|c|}
\hline Factor $\mathrm{T}$ & ype & I & evels & Talues & & & & \\
\hline Amina & ixed & & 3 & $0 ; 10 ; 2$ & & & & \\
\hline Amido & ixed & & 3 & $0 ; 200 ;$ & 00 & & & \\
\hline ysis & f & $\operatorname{Tari}$ & ance for & Fe rej & (s), usi & $\mathrm{ng} \mathrm{Adj}$ & sted & Eor Tests \\
\hline Source & & $\mathrm{DF}$ & Seq SS & Adj SS & Adj MS & $\mathrm{F}$ & $\mathrm{P}$ & \\
\hline Amina & & 2 & 0,0051 & 0,0051 & 0,0026 & 0,02 & 0,980 & \\
\hline Amido & & 2 & 0,1709 & 0,1709 & 0,0855 & 0,66 & 0,538 & \\
\hline Amina $*$ Amic & do & 4 & 0,7713 & 0,7713 & 0,1928 & 1,50 & 0,281 & \\
\hline Error & & 9 & 1,1570 & 1,1570 & 0,1286 & & & \\
\hline Total & & 17 & 2,1043 & & & & & \\
\hline
\end{tabular}

Figura 5: Análise estatística do teor de ferro no rejeito.

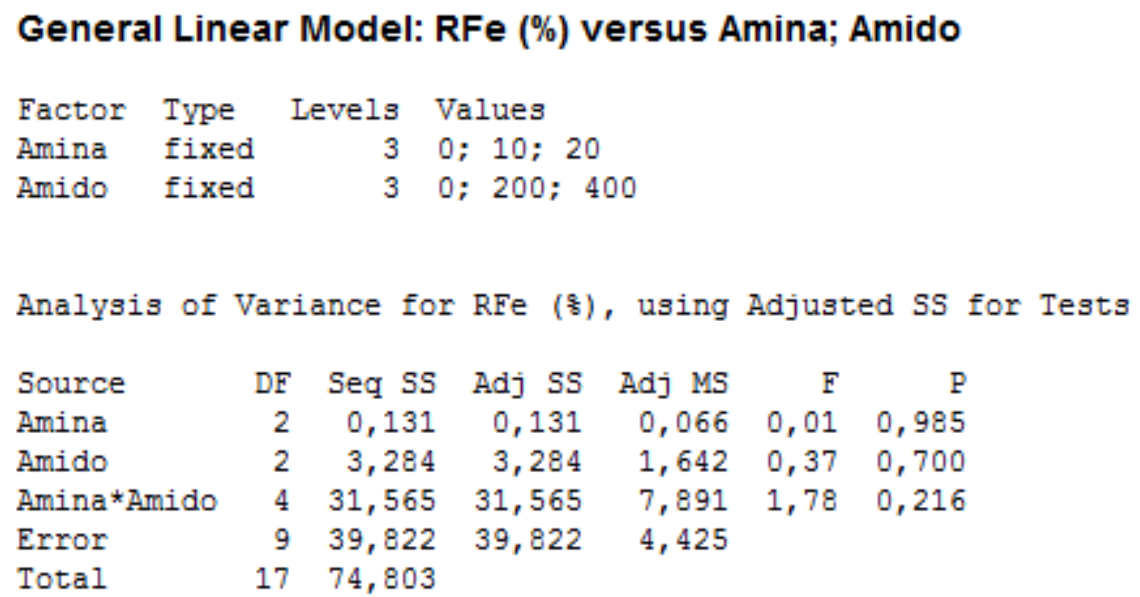

Figura 6: Análise estatística do índice de recuperação metalúrgica.

\section{CONCLUSÃO}

Os resultados dos testes laboratoriais mostraram que a flotação do overflow da etapa rougher, que atualmente não é reaproveitado, proporcionou uma redução significativa dos teores de ferro no rejeito, em torno de $62 \%$, fato que corresponde a um índice de recuperação metálica de $78 \%$. Além disso, os testes estatísticos demonstraram não ser necessária a dosagem suplementar de reagentes para flotar este material.

\section{REFERÊNCIAS}

1 MONTGOMERY, Douglas C.Applied. Statistics and probability for engineers / Douglas C. Montgomery, George C.Runger. 3th ed. Arizona: John Wiley \& Sons, Inc, 2008. 